\title{
Magnetic Heating by Tunable Arrays of Nanoparticles in Cancer Therapy
}

\author{
V. ZablotskiI ${ }^{a, b, *}$, O. Lunov ${ }^{c}$ And C. Gómez-Polo ${ }^{a}$ \\ ${ }^{a}$ Departamento de Fisica, Universidad Publica de Navarra \\ 31006, Pamplona, Spain \\ ${ }^{b}$ Institute of Experimental Physics, University of Białystok \\ Lipowa 41, 15-424 Białystok, Poland \\ ${ }^{c}$ Institute of Pharmacology of Natural Products \& Clinical Pharmacology \\ University of Ulm, Helmholtzstr. 20, D-89081 Ulm, Germany
}

\begin{abstract}
Detailed knowledge about the temperature distribution achieved in the target area is essential for the development of magnetic hyperthermia treatments. However, the temperature inhomogeneity was found in all local hyperthermia studies. As a consequence of the impossibility of guaranteeing the temperature and thus the thermal dose distribution, hyperthermia is never applied as a single treatment modality. We suggest a model that enables the calculations and optimization of the spatial-time distribution of the temperature in the target volume (i.e. tumor) caused by magnetically heated elements: (i) arrays of clusters of iron oxides magnetite $\left(\mathrm{Fe}_{3} \mathrm{O}_{4}\right)$ magnetic nanoparticles, and (ii) arrays of magnetic needles. In order to find the spatial-time temperature distribution in tumor, the bioheat transfer equation is solved for the two above-mentioned arrays of magnetically heated sources embedded in the tumor. The temporal and spatial temperature distributions were calculated with regard to the effect of blood perfusion in the tumor. It is shown that a matrix of magnetic micro-needles injected in the tumor could provide rather uniform tumor heating with the center-edge temperature difference smaller than $3^{\circ} \mathrm{C}$ at any times during the magnetic hyperthermia treatments. The temperature profiles can be suitably adjusted by a proper choice of the magnetic nanoparticles arrangement.
\end{abstract}

PACS numbers: 87.55.D-, 87.55.de, 89.20.-a, 75.50.Tt

\section{Introduction}

Localized hyperthermia utilizing magnetic nanoparticles (MNPs) in an alternating magnetic field has the potential to either directly kill tumors or make them more susceptible to radiation therapy or chemotherapy [1-4]. The iron oxides magnetite $\left(\mathrm{Fe}_{3} \mathrm{O}_{4}\right)$ and maghemite $\left(\gamma-\mathrm{Fe}_{2} \mathrm{O}_{3}\right)$ nanoparticles are the most studied to date because of their generally appropriate magnetic properties and biological compatibility [5].

The heating effect strongly depends on the size, shape, and magnetic permeability of the nanoparticles and on the frequency and amplitude of the applied alternating magnetic field [6-10]. Numerical and analytical studies [11-14] of the heating effect of MNPs embedded in tumor showed that in most cases of local hyperthermia treatments, temperature inhomogeneities were found. Nevertheless, to achieve the therapeutic effect a minimal temperature of $43^{\circ} \mathrm{C}$ is typically required in the whole tumor area. Looking for the ways to improve the homogeneity of the temperature distribution in a tumor, in this work we analyze the temperature profiles for two partic-

\footnotetext{
* corresponding author; e-mail: zablot@fzu.cz
}

ular cases: (i) clusters of MNPs dispersed in tumor and (ii) square arrays of micron sized rods (needles) of MNPs embedded in tumor.

We present a model to determine the time and spatial distribution of temperature in a tumor and its dependence on the blood perfusion as well as on the power dissipation of a MNP. In terms of this model the MNPs heating for magnetic hyperthermia treatments can be optimized to achieve the therapeutic effect in the whole treated area. The model is particularized for the time and spatial temperature distributions produced by iron oxide magnetite $\left(\mathrm{Fe}_{3} \mathrm{O}_{4}\right)$ nanoparticles embedded in a tumor.

\section{Model}

It is known that hyperthermia cancer treatment kills cancerous cells by elevating their temperatures to the therapeutic temperature range, $42-45^{\circ} \mathrm{C}$. This approach can destroy tumors with minimal damage to healthy tissues and, therefore, limit negative side effects $[1,2]$. The mentioned temperature range determines the upper limit of the degree of the spatial non-homogeneous temperature distribution as $\delta T=2-3^{\circ} \mathrm{C}$. Therefore, the temper- 
atures taken in the tumor center and close to its edge should differ less than $\delta T$ for any time of the hyperthermia treatment.

Let us analyze the time and spatial distribution produced by clusters of MNPs embedded in tumor. In tissue spatial clusters of MNPs could appear as a result of biodistributions (biological processes in a tumor). Moreover, to achieve the needed temperatures one should increase the concentration of MNPs up to $n=10^{21-22} \mathrm{~m}^{-3}$ $[3,11]$. Such a high concentration favors a clustering of MNPs to minimize their magnetic dipoles energy. From a practical point of view the mean period of the cluster structure could be more easily adjusted for the purposes of turning temperature profiles than that in the case of single MNPs dispersed in a tumor. Thus, it can be assumed that a cluster structure of MNPs appears, for example, as a result of the biodistribution of MNPs in tumors [15]. To describe the cluster structure of MNPs we introduce two parameters: $R_{\mathrm{cl}}$, the cluster radius, and $p$, the mean spatial period of clusters.

In a tumor the temperature distributions, $T(x, y, z, t)$ could be described by the so-called bioheat transfer equation $[16]$ :

$$
\rho c_{v} \frac{\partial T}{\partial t}=k \Delta T+c w \rho_{\mathrm{b}}\left(T_{0}-T\right)+\sum_{i=1}^{N} q\left(x_{i}, y_{i}, z_{i}\right),
$$

where $\Delta$ is the Laplace operator, $\rho$ is the density of tissue, $c_{v}$ is the heat capacity of tissue, $k$ is the thermal conductivity coefficient of tissue, $w$ is the blood perfusion (in general, $w$ could be coordinate dependent reflecting a specific vessel tree in tumor), $c$ and $\rho_{\mathrm{b}}$ are the heat capacity and mass density of blood, $T_{0}$ is the temperature of blood (which was taken as $37^{\circ} \mathrm{C}$ ), $q$ is the volumetric heat power in a cluster; $N$ is the total number of clusters nanoparticles, and $x_{i}, y_{i}$, and $z_{i}$ are the coordinates of clusters location. Let us notice that for the case of a coordinate independent $q$ (the case $q=$ const corresponds to the same heat power dissipation in each point of the heated area) and neglecting the blood perfusion term, the analytical solution of Eq. (1) was done in [13]. The blood perfusion is defined as the ratio of the volumetric flow rate of the blood $\left(=\pi r_{\mathrm{b}}^{2} v\right)$ and the volume of the tumor [17] (where $v$ is the linear blood velocity and $r_{\mathrm{b}}$ is the radius of blood vessels in the tumor). Further calculations of temporal and spatial temperature distributions will be done for the two values of the blood perfusion rate: $w=2.6 \times 10^{-4} \mathrm{~s}^{-1}$ and $w=5.2 \times 10^{-4} \mathrm{~s}^{-1}$, to which for a tumor with the geometrical parameters $1 \times 2 \times 3 \mathrm{~cm}^{3}$ and $r_{\mathrm{b}}=10^{-2} \mathrm{~cm}$ (typical of lung cancer Stage 0, Stage IA, Stage IIA, occult carcinoma [18]) correspond the blood velocities $v=5 \mathrm{~cm} / \mathrm{s}$ and $10 \mathrm{~cm} / \mathrm{s}$. As a function of the MNP and medium parameters and applied magnetic field, the volumetric power dissipation, $q$, was determined in [6].

For common types of MNP the specific heating power is about $100 \mathrm{~W} / \mathrm{g}$ [2] which for $\mathrm{Fe}_{3} \mathrm{O}_{4}$ MNPs corresponds to $q=7.874 \times 10^{8} \mathrm{~W} / \mathrm{m}^{3}$. This value of $q$ will be used in our further calculations. A higher value of the specific heating power can be achieved for example for cobaltferrite MNPs [19] (440 W/g, at frequency and amplitude of the ac magnetic field of $700 \mathrm{kHz}$ of $31 \mathrm{mT}$, respectively).

Solving numerically Eq. (1), with the physical parameters of cancer tissue taken from literature [2, 17, 20-25], one can obtain the spatial and time distribution of temperature in the tumor, $T(x, y, z, t)$. For simplicity and clearness in the presentation of the calculated results, first, we consider 2D temperature distributions in the tumor with a periodic cluster structure of MNPs.

\section{Temperature distributions in tissue heated by a cluster structure of MNPs}

For the 2D case Eq. (1) was numerically solved with the following imposed initial and boundary conditions: $T(x, y, 0)=37^{\circ} \mathrm{C}$ (temperature of human heat core) at $t=0$, and equality of the heat fluxes at the interface between the tumor and normal tissue, $k_{\mathrm{n}} \mathrm{d} T / \mathrm{d} \xi=k \mathrm{~d} T / \mathrm{d} \xi$, where $k_{\mathrm{n}}$ and $k$ are the thermal conductivity coefficients of normal tissue and tumor, accordingly; here $\xi$ denotes the normal coordinate to the interface. Numerical solutions were obtained with the help of the software package MathCad 13, with the following parameters: $c_{v} \approx 5.136 \mathrm{~kJ} /(\mathrm{kg} \mathrm{K})[23,26] ; k \approx 0.64 \mathrm{~W} / \mathrm{mK}[2]$ and $k_{\mathrm{n}}=0.48 \mathrm{~W} / \mathrm{mK}, \rho \approx 800 \mathrm{~kg} / \mathrm{m}^{3}, c=3.594 \mathrm{~kJ} /(\mathrm{kg} \mathrm{K})$ [23], $R_{\mathrm{cl}}=10 \mu \mathrm{m}, R=10 \mathrm{~nm}$ is the particle radius, $p=220 \mu \mathrm{m}$, and $N=2000$ is the number of clusters in the tumor of size $1 \times 1 \mathrm{~cm}^{2}$. The chosen mean cluster radius and period correspond to the MNPs fraction $50 \mathrm{mg}$ of MNPs per $1 \mathrm{~kg}$ of tissue - which was experimentally approved for the effective therapy in [24, 25, 27]. Let us notice that we assume that in tissue MNPs were injected in a vein and distributed in tumor without guidance. MNPs clustering was reported in [15], where $10 \mathrm{~nm}$ sized MNPs created a disordered cluster structure with the mean period about $200 \mu \mathrm{m}$. The calculations were made for two values of blood perfusion characterized by linear blood velocity values, $v=5 \mathrm{~cm} / \mathrm{s}$ and $10 \mathrm{~cm} / \mathrm{s}$.

Figure 1 shows the contour plots of the spatial temperature distributions $\left(\Delta T=T-T_{0}\right.$, where $T_{0}$ is the initial temperature) at two different times after switching on the external alternating magnetic field assuming a homogeneous perfusion with mean blood velocity $v=5 \mathrm{~cm} / \mathrm{s}$.

As Fig. 1 shows, the temperature increases from the external area of the tumor to the central part of it. In fact, it is seen that the clusters of MNPs embedded in the tumor produce nonuniform temperature profiles resembling those obtained in [13] under the assumption of an independent coordinate $q$ and neglecting the blood perfusion term.

With respect to the influence of the blood velocity, the temperature distribution in the same tumor area was calculated for different values of the blood velocity (see Fig. 2). As it is seen from Fig. 2, the spatial rate of the temperature increase in the tumor is strongly dependent on the blood velocity. The rate of the temperature increase is lower for higher values of the blood velocity. 


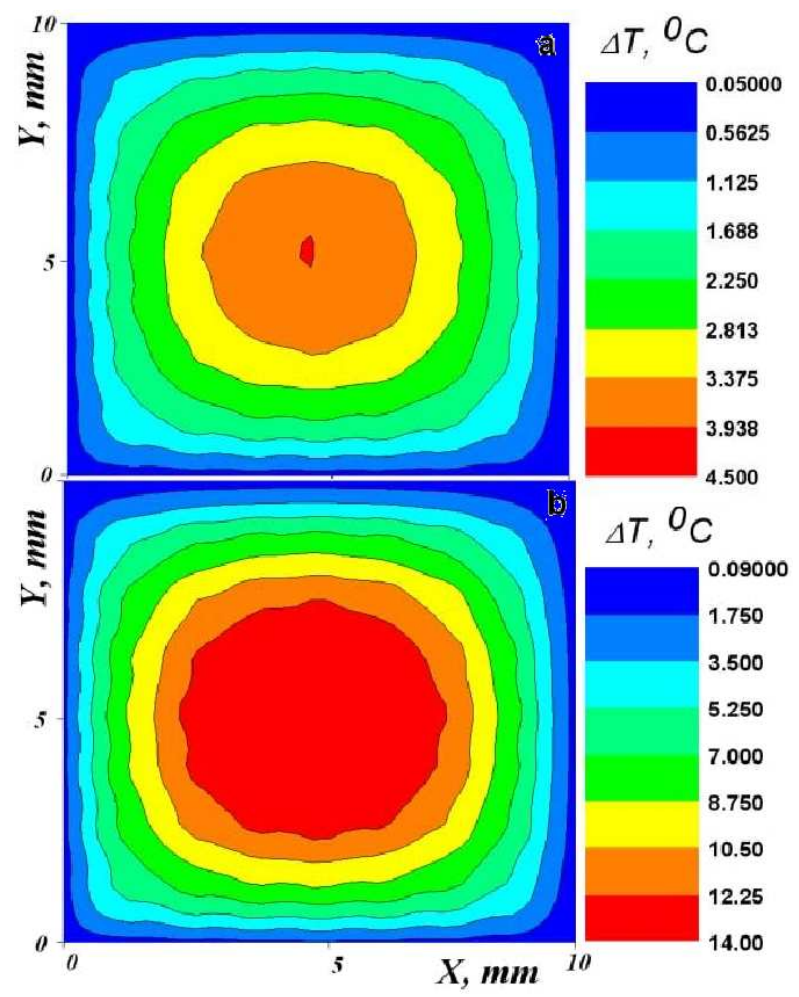

Fig. 1. Temperature distributions in a tumor with $N=2000$ and blood velocity $v=5 \mathrm{~cm} / \mathrm{s}$ : (a) at $3 \mathrm{~min}$ and (b) at 10 min after switching on external alternating magnetic field.

We now estimate the influence of blood vessels on the temperature increase during hyperthermia treatments. As seen from Figs. $2 \mathrm{a}-\mathrm{c}$, homogeneous blood perfusion lowers temperature $(\Delta T(x, y)$ taken in the central part of the tumor) by $1^{\circ} \mathrm{C}$ and $4^{\circ} \mathrm{C}$, for $v=5 \mathrm{~cm} / \mathrm{s}$ and $v=10 \mathrm{~cm} / \mathrm{s}$, accordingly. Obviously, the same temperature decrease could be expected in the vicinity of large blood vessels. Indeed, a number of cold spots with temperature fault about $2-4^{\circ} \mathrm{C}$ was found in the vicinity the large vessels during interstitial hyperthermia using ferromagnetic seeds (inter-seeds spacing $13 \mathrm{~mm}$ ) in [28]. Tumor blood perfusion could be adjusted with the goal to obtain the needed uniformity and rate of temperature increase in hyperthermia treatments.

Thus, heating with a cluster structure of MNPs gives rather non-uniform temperature distributions similar to those obtained for MNPs uniformly dispersed in the tumor [13] or localized in the small (millimeter-sized) areas of the tumor $[11,12]$. However, there are two points which determine the value of the obtained results. First, we have proved that in spite of the fact that a MNP (or MNPs cluster) is highly focused heat source, the commonly used assumption on uniformly distributed heat in tissue is good enough for a description of the temperature profiles and their time evolution. Second, the results displayed in Figs. 1 and 2 show how to adjust the clusters distribution in order to make a more uni-

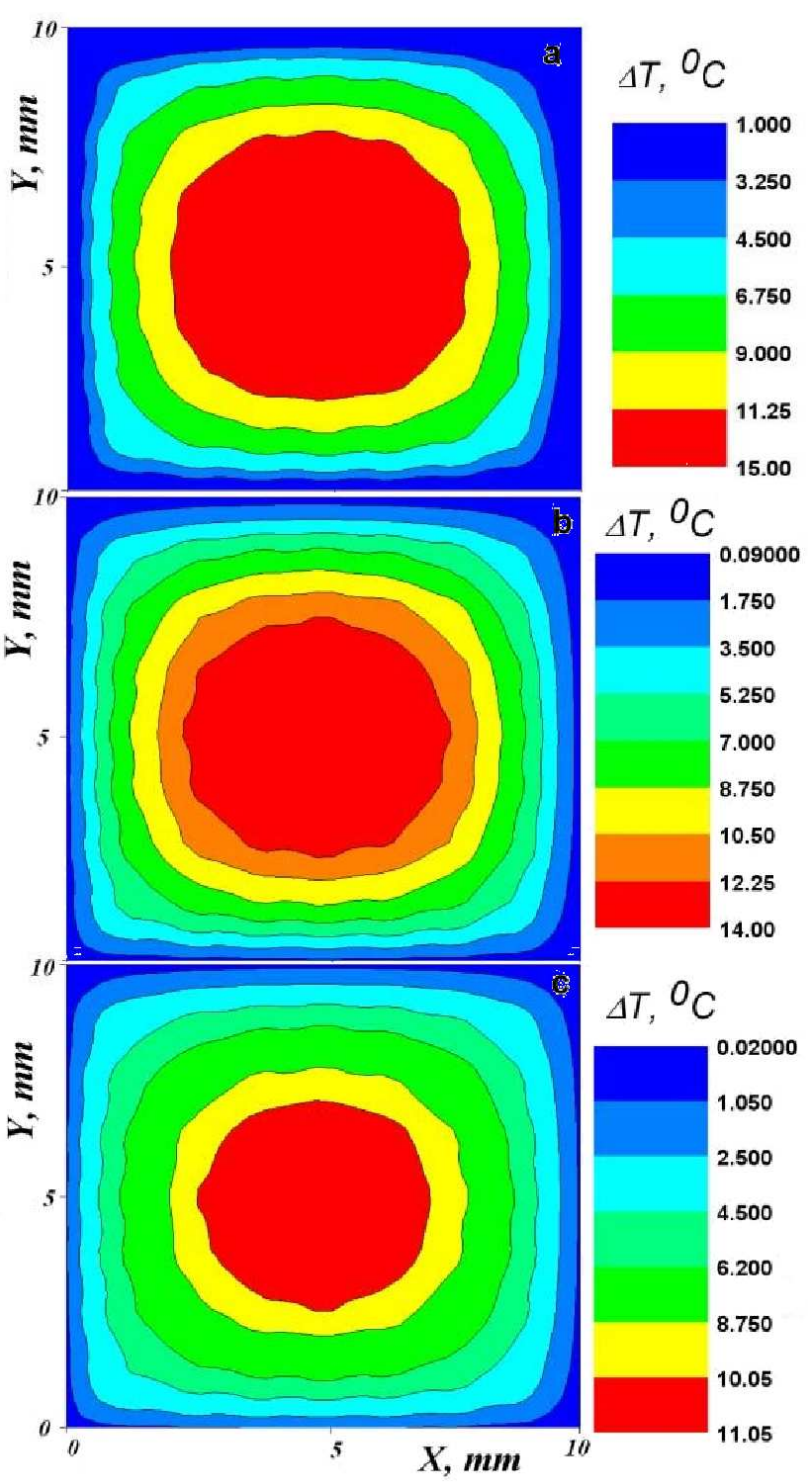

Fig. 2. Temperature distributions in a tumor area with $N=2000$ at 10 min after switching on external alternating magnetic field for blood velocities: (a) $v=0$; (b) $v=5 \mathrm{~cm} / \mathrm{s}$, and (c) $v=10 \mathrm{~cm} / \mathrm{s}$.

form temperature distribution. Indeed, at the distance about $1 \mathrm{~mm}$ from the tumor edge the temperature decrease (in comparison with that in the tumor center) is about $5^{\circ} \mathrm{C}$. Knowing that the temperature is proportional to the heat source density (see for instance Eq. (2)) one could improve the temperature profile homogeneously by a proper decrease in the cluster period in the tumor edge area. In the next section we suggest another type of MNPs distribution leading to quite uniform temperature profiles.

\section{Heating with a square array of micro-needles}

Let us consider a square array of heating micro-needles with MNPs inside embedded in tumor. We assume that 
the micro-needles are parallel to the $z$-axis. For this geometry and zero initial conditions, neglecting the blood perfusion term, Eq. (1) allows the analytical solution [29]:

$$
\begin{aligned}
& \Delta T(x, y, t)=\frac{\bar{Q}}{c_{v} \rho} \int_{0}^{t} \sum_{i=1}^{N_{1}} \sum_{j=1}^{N_{1}}\left(\frac{1}{2 a \sqrt{\pi t}}\right)^{2} \\
& \times \exp \left(\frac{\left(x-x_{i}\right)^{2}+\left(y-y_{j}\right)^{2}}{4 a^{2} t}\right) \mathrm{d} t
\end{aligned}
$$

where $a^{2}=k / c_{v} \rho, \Delta T$ is the temperature increase, $\bar{Q}$ is the linear power dissipation, i.e. $\bar{Q}=q \pi R_{\mathrm{w}}^{2}, R_{\mathrm{w}}$ is the rod radius, and the total number of rods is $N=N_{1} \times N_{1}$. In principal, the heating elements could be homogeneous ferromagnetic rods. However, most real ferromagnetic materials require a big enough applied field strength ( $c a$. $100 \mathrm{kA} / \mathrm{m}$ ) to achieve high values of the specific absorption rate [5]. In contrast, MNPs are capable to generate sufficient heating at lower magnetic fields due to their particular relaxation processes.

Using Eq. (2), the calculations of $T(x, y, t)$ were performed for a square tumor of size $3.0 \times 3.0 \mathrm{~cm}^{2}$ with $N=300 \times 300$ needles of radius $R_{\mathrm{w}}=1.0 \mu \mathrm{m}$ and the period $p=100 \mu \mathrm{m}$. The filling fraction $30 \%$ of NMPs inside a needle was assumed. The results of calculations are shown in Fig. 3.

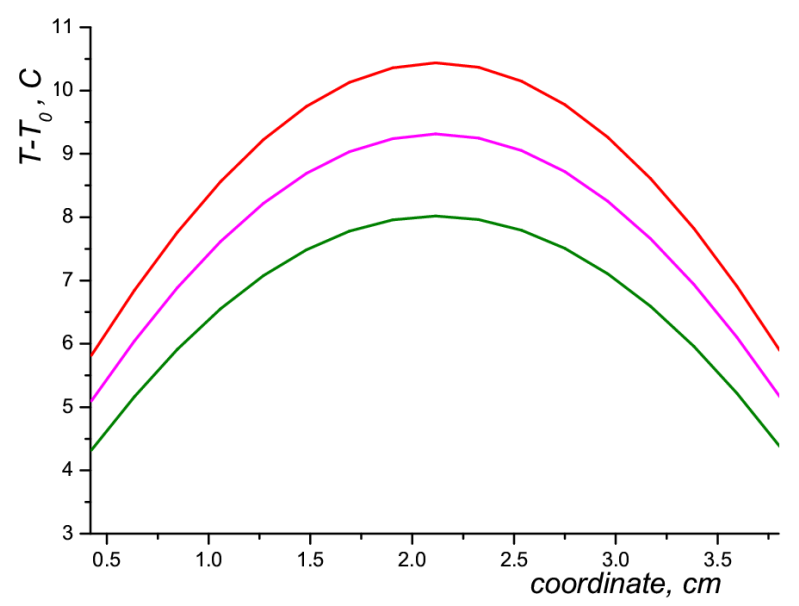

Fig. 3. Temperature profiles calculated along the diagonal of the tumor at different times (from bottom to top): $t_{0}=400 \mathrm{~s}, t_{0}=500 \mathrm{~s}$, and $t_{0}=600 \mathrm{~s}$.

Figure 3 shows the temperature profiles calculated along the diagonal of the tumor at different times: $t_{0}=$ $400 \mathrm{~s}, t_{0}=500 \mathrm{~s}$, and $t_{0}=600 \mathrm{~s}$ after switching on the applied magnetic field. It is seen from Fig. 3 that, in the central part of tumor (which is $2 \times 2 \mathrm{~cm}^{2}$ for the tumor of $3 \times 3 \mathrm{~cm}^{2}$ ), the periodical array of microtubes filled up by MNPs produces the temperature profiles with the degree of homogeneity smaller than $\delta T=3^{\circ} \mathrm{C}$. It is important to note that the profiles are convex ones and there are no relatively low temperatures further away from the magnetic needles similarly, as it was found in the hyperthermia treatments using macroscopic ferromagnetic seeds in [28].
From a practical point of view a matrix of micro-needles with MNPs inside could be easily injected in a tumor and heated by ac field of suitable amplitude and frequency. Different types of matrices could be designed for specific tumors. Using a matrix of needles filled by MNPs for practical hyperthermia one can obtain the needed temperature profile and heating time adjusting the combination of the parameters: $R_{\mathrm{w}}, p$, and the filling fraction.

\section{Conclusions and discussion}

A model allowing calculations of temperature distributions in a tumor and its dependence on physical parameters of the tissue has been proposed. We calculated the temperature distributions in tumors with two variants of nanoparticles' location: cluster structure of MNPs and a square array of needles (with MNPs inside). It was found that exposed to a high-frequency magnetic field and dispersed in the tumor with the mean spatial period $200 \mu \mathrm{m}$ clusters of $\mathrm{Fe}_{3} \mathrm{O}_{4}$ nanoparticles can produce temperature profiles allowing to achieve the therapeutic effect only in the central area of tumor. It is also shown that the rate of the temperature increase and the temperature homogeneity could be tuned by a proper change of the blood perfusion.

To improve the spatial temperature homogeneity we have suggested a new system of micro-heating elements - the matrix of micro-tubes containing MNPs and injected in tumor. As an example, by analytical calculations, we show that such a system could provide rather uniform tumor heating with the center-edge temperature difference smaller that $3^{\circ} \mathrm{C}$ at any time during the magnetic hyperthermia treatments. This allows us to achieve the therapeutic effect over the whole tumor region.

\section{Acknowledgments}

This work was performed within the framework of the NANORET project (Interreg III A Francia-España 2000-2006). V. Zablotskii acknowledges the financial support for the sabbatical staying at the Dpto. Fisica, Universidad Pública de Navarra from the Spanish Ministry of Education and Science (SAB2006-0120). This work was also supported by the Marie Curie Host Fellowships for Transfer of Knowledge - the NANOMAG-LAB project (2004-003177).

\section{References}

[1] R. Hergt, W. Andra, C.G. d'Ambly, I. Hilger, W.A. Kaiser, U. Richter, H.-G. Schmidt, IEEE Trans. Magn. 34, 3745 (1998).

[2] R. Hergt, S. Dutz, J. Magn. Magn. Mater. 311, 187 (2007).

[3] A. Jordan, R. Scholz, P. Wust, H. Schirra, T. Schiestel, H. Schmidt, R. Felix, J. Magn. Magn. Mater. 194, 185 (1999). 
[4] J.J.W. Lagendijk, Phys. Med. Biol. 45, R61 (2000).

[5] Q.A. Pankhurst, J. Connolly, S.K. Jones, J. Dobson, J. Phys. D, Appl. Phys. 36, R167 (2003).

[6] R.E. Rosensweig, J. Magn. Magn. Mater. 252, 370 (2002).

[7] I. Baker, Q. Zeng, W. Li, C.R. Sullivan, J. Appl. Phys. 99, $08 \mathrm{H} 106$ (2006).

[8] S. Maenosono, S. Saita, IEEE Trans. Magn. 42, 1638 (2006).

[9] R. Hergt, S. Dutz, R. Muller, M. Zeisberger, J. Phys., Condens. Matter 18, S2919 (2006).

[10] M. Veverka, P. Veverka, O. Kaman, A. Lancok, K. Zaveta, E. Pollert, K. Knizek, J. Bohacek, M. Benes, P. Kaspar, E. Duguetand, S. Vasseur, Nanotechnology 18, 345704 (2007).

[11] Y.G. Lv, Z-S. Deng, J. Liu, IEEE Trans. Nanobiosci. 4, 284 (2005).

[12] W. Andra, C.G. d'Ambly, R. Hergt, I. Hilger, W.A. Kaiser, J. Magn. Magn. Mater. 194, 197 (1999).

[13] Y. Rabin, Int. J. Hyperthermia 18, 194 (2002).

[14] R. Xu, Y. Zhang, M. Ma, J. Xia, J. Liu, N. Gu, IEEE Trans. Magn. 43, 1078 (2007).

[15] O. Brunke, S. Odenbach, C. Fritsche, I. Hilger, W.A. Kaiser, J. Magn. Magn. Mater. 289, 428 (2005).

[16] H.H. Pennes, J. Appl. Physiol. 1, 93 (1948).

[17] A.C. Guyton, J.E. Hall, Ph.D. Textbook of Medical Physiology, 10th ed., W.B. Saunders Company, A Harcourt Health Company, Philadelphia 2000, p. 25.
[18] F. Clifton, Chest 111, 1710 (1997).

[19] J.-P. Fortin, F. Gazeau, C. Wilhelm, Eur. Biophys. J. 37, 223 (2007).

[20] A. Wijaya, K.A. Brown, J.D. Alper, K. Hamad-Schifferli, J. Magn. Magn. Mater. 309, 15 (2007).

[21] M. Soehle, M. Czosnyka, J.D. Pickard, P.J. Kirkpatrick, Stroke 35, 1393 (2004).

[22] R. Aaslid, S.R. Lash, G.H. Bardy, W.H. Gild, D.W. Newell, Stroke 34, 1645 (2003).

[23] E. Ponder, J. General Physiol. 45, 545 (1962).

[24] F. Matsuoka, M. Shinkai, H. Honda, T. Kubo, T. Sugita, T. Kobayashi, BioMagnetic Res. Technol. 2, 1 (2004).

[25] K. Tanaka, T. Ito, T. Kobayashi, T. Kawamura, S. Shimada, K. Matsumoto, T. Saida, H. Honda, J. Biosci. Bioeng. 100, 112 (2005).

[26] S.R. Upreti, A.A. Jeje, Chem. Eng. Sci. 59, 4415 (2004).

[27] A. Ito, K. Tanaka, K. Kondo, M. Shinkai, H. Honda, K. Matsumoto, T. Saida, T. Kobayashi, Cancer Sci. 93, 308 (2003).

[28] N. Wieringenyz, A.N. Kottey, G.M.J. Leeuweny, J.J.W. Lagendijky, J.D.P. Dijkx, G.J. Nieuwenhuys, Phys. Med. Biol. 43, 121 (1998).

[29] A.N. Tichonov, A.A. Samarskii, Equations of Mathematical Physics, Nauka, Moscow 1977. 